\begin{tabular}{|l|l|l|l|}
\hline Eiszeitalter u. Gegenwart & $\mathbf{3 0}$ & $\begin{array}{c}101-108 \\
2 \mathrm{Abb}, 5 \mathrm{Tab} .\end{array}$ & Hannover 1980 \\
\hline
\end{tabular}

\title{
Periglaziale Deckschichten auf weichselzeitlichen Sedimenten in Polen
}

\begin{abstract}
Arno Semmel *)
Periglacial, podzol, stratification, particle size analysis, heavy mineral, trace element analysis, Weichselian glaciation, Poland

Kurzfassung: Aus dem Gebiet der weichselzeitlichen Vereisung in Polen werden allgemein verbreitete geringmächtige periglaziale Deckschichten beschrieben. Sie zeigen in der Regel eine äolische Beeinflussung und unterscheiden sich dadurch vom Liegenden. Es handelt sich um spätglaziale Bildungen, wie sie aus dem Jungmoränengebiet der DDR seit langem bekannt sind. Ähnliche Substrate wurden auch im nördlichen Alpenvorland gefunden.
\end{abstract}

\section{[Periglacial Cover Layers on Weichselian-Sediments in Poland]}

Abstract: From the area of the Weichselian glaciation in Poland, wide-spread, thin periglacial cover-layers are described. They possess, as a rule, an acolian component, and can thus be distinguished from underlying strata. They are of Late-Glacial origin, and are similar to strata, which have long been known from the Weichselian area of the German Democratic Republic. Similar strata have also been found in the northern Alpine Foreland.

\section{Einleitung}

Untersuchungsbefunde über spätglaziale Deckschichten oder „Umlagerungszonen “ auf Sedimenten der letzten Eiszeit in Mitteleuropa sind bereits wiederholt publiziert worden. Vor allem mit der Arbeit von Kopp (1965) ist eine Diskussion der Bedeutung solcher Sedimente für die Bodenentwicklung auf Moränen und anderen glazigenen Sedimenten der letzten Eiszeit eröffnet worden, die bis heute anhält (Diemann 1977). In jüngster Zeit berichten auch BLUME et al. (1978) von der kryoturbaten Vorprägung in Parabraunerdeprofilen auf Moränenplatten in der Umgebung Berlins. Von entsprechenden Erscheinungen in der Jungmoränenlandschaft Schleswig-Holsteins liegen bisher kaum Angaben vor (SEMMEL 1973: 119).

Im nördlichen Alpenvorland sind dagegen ohne Zweifel entsprechende Bildungen weit verbreitet (Semmel 1973). Reisen in Polen boten Gelegenheit, der Frage nachzugehen, ob auf den dortigen Ablagerungen der Weichsel-Eiszeit ebenfalls periglaziale Deckschichten entwickelt sind und ob diese auf den Moränen der verschiedenen Stadien unterschiedliche Ausprägung zeigen.

Die Reiserouten wurden so gewählt, daß jeweils die gesamte Moränenfolge zwischen den Sudeten und der Ostsee gequert und in der Regel die von der Straße her zugänglichen Aufschlüsse untersucht werden konnten (vgl. Abb. 1). Darüber hinaus sind in manchen Gebieten zusätzliche Untersuchungen in abseits der Durchgangsstraßen liegenden Aufschlüssen vorgenommen worden. Das gilt für die Umgebung von Mlawa (nördlich Warschau), für das Gebiet um Chojna (Königsberg/Neumark) und Zielona Gora (Grünberg).

*) Anschrift des Verfassers: Prof. Dr. A. S e m me 1, Institut für Physische Geographie der Universität, Senckenberganlage 36, D-6000 Frankfurt am Main. 


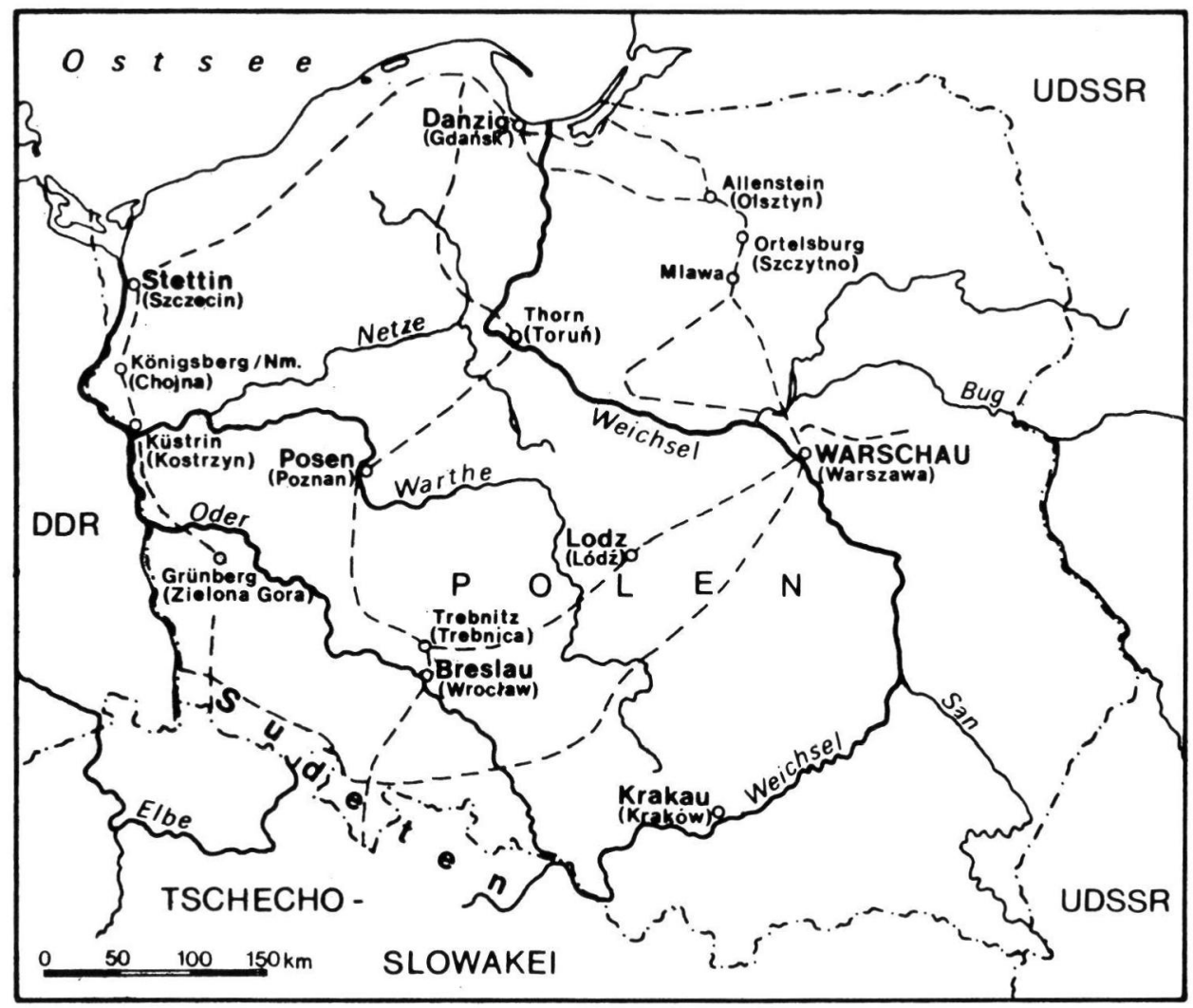

-- Reiserouten

Abb. 1: Ubersichtsskizze der Reiserouten.

Dabei ergab sich, daß auf fast allen aufgeschlossenen glazigenen Bildungen der Weichsel-Eiszeit eine jüngere, manchmal mehrgliedrige Deckschicht liegt. Nur dort, wo starke Beackerung mit Bodenerosion verbunden war, fehlt eine solche Lage. Anhand ausgewählter Beispiele werden im folgenden Aufbau und Eigenschaften solcher Deckschichten näher erläutert. Die stratigraphische Einstufung der liegenden glazigenen Substrate erfolgt nach den Angaben von LiedTke (1975).

\section{Aufbau und Eigenschaften von Deckschichten auf glazigenen Sedimenten der Weichsel-Eiszeit}

Auf relativ engem Raum liegen die Bildungen des Brandenburger, des Frankfurter und des Pommerschen Stadiums zwischen Mlawa und Olsztyn (Allenstein) beieinander. In mehreren Aufschlüssen südöstlich von Allenstein, östlich der von Szczytno (Ortelsburg) kommenden Straße, ist die kuppige Grundmoräne des Pommerschen Stadiums aufgeschlossen. In Richtung Ortelsburg schließen Aufschlüsse in den Endmoränen und Sandern dieses Stadiums an. Allen gemeinsam ist, daß in keinem Fall die glazigenen oder glazifluvialen Sedimente direkt unter der Oberfläche liegen, sondern von einer sandigen Deckschicht 
überzogen werden, die überwiegend 40 bis $70 \mathrm{~cm}$ mächtig ist. So liegt z. B. am Südrand von Allenstein im Aufschluß am See unmittelbar östlich der Straße nach Ortelsburg unter einem mit $5^{\circ}$ nach Süden geneigten, mit Kiefern bestandenen Hang folgendes Profil:

$3 \mathrm{~cm}$ organische Auflage (Rohhumus)

\section{0 - $2 \mathrm{~cm}$ Podsol-Bleichhorizont $\left(\mathrm{A}_{\mathrm{e}}\right)$}

2- $40 \mathrm{~cm}$ brauner, schluffig-lehmiger Sand $\left(B_{v}\right)$ mit hangabwärts eingeregelten Geröllen, an der Basis eine Lage von Geröllen, in Hangrichtung eingeregelt, einzelne etwas aufgerichtet

$40-50 \mathrm{~cm}$ fahl-hellbrauner, schwach schluffiger Sand (II $A_{1}$ )

$50-80 \mathrm{~cm}$ rostbrauner, toniger Sand (II B $\left.{ }_{t}\right)$

$80-150 \mathrm{~cm}+$ hellbrauner Sand, geschichtet, mit rostbraunen, tonigen Bändern.

In diesem Profil zeigt die hangparallel verlaufende Steinlage bei $40 \mathrm{~cm}$ eine Grenze an, die nicht als Folge der Bodenbildung gedeutet werden kann, obwohl sie gleichzeitig die Grenze des $B_{v}$-Horizontes darstellt: Die Einregelung der einzelnen Steine in Gefällsrichtung deutet auf eine solifluidale Bewegung im wasserreichen Auftauboden hin, die wahrscheinlich über gefrorenem Untergrund stattgefunden hat, der zugleich ein Einsinken der Steine in den tieferen Sand verhinderte (vgl. hierzu auch Kopp 1970: $274 \mathrm{ff}$.; Kopp \& JäGer 1972; Semmel 1969; 42 ff.). Will man diesen Vorgang als holozänes Phänomen deuten, so bleiben die Schwierigkeiten zu erklären, die auf S. 105 noch näher erörtert werden.

Im Gegensatz zum Liegenden besitzt die Solifluidalschicht meist eine abweichende Korngrößenzusammensetzung. Das Profil bei Allenstein weist einen geringeren Sandund einen höheren Schluffgehalt oberhalb der Steinlage auf (vgl. Tab. 1). Das kann nicht die Auswirkung einer in situ-Verwitterung sein, denn mit diesen Korngrößenunterschieden ist eine Veränderung in der Schwermineralführung verbunden (Tab. 2). Der Schluff enthält im Gegensatz zum Sand eine „vulkanisch-äolische“ Komponente (Augit und braune Hornblende). Damit ist eine Differenzierung gegeben, wie sie etwa der tundrenzeitliche Deckschutt (Semmel 1964, 1968) in den hessischen Mittelgebirgen häufig gegen-

Tab. 1: Korngrößen (in \%) des Profils südlich Allenstein (Olsztyn)

\begin{tabular}{rccccccc}
\hline Flurabstand & $<0,002$ & $0,002-0,006$ & $0,006-0,02$ & $0,02-0,06$ & $0,06-0,2$ & $0,2-0,6$ & $0,6-2 \mathrm{~mm} \phi$ \\
\hline $2-40 \mathrm{~cm}$ & 6,3 & 6,1 & 14,3 & 25,9 & 11,5 & 21,3 & 9,7 \\
$40-50 \mathrm{~cm}$ & 3,7 & 2,7 & 3,6 & 3,9 & 24,3 & 45,7 & 11,7 \\
$50-80 \mathrm{~cm}$ & 9,3 & 1,1 & 2,7 & 3,0 & 24,1 & 46,5 & 9,2
\end{tabular}

Tab. 2: Schwermineralführung in \% im Profil südlich Allenstein (Olsztyn)

\begin{tabular}{|c|c|c|c|c|c|c|c|c|c|c|c|c|c|c|}
\hline Flurabstand & $\begin{array}{l}\frac{x}{\pi} \\
\text { مू }\end{array}$ & 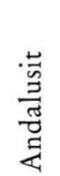 & $\begin{array}{l}\text { · } \\
\text { 尊 }\end{array}$ & 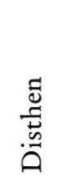 & 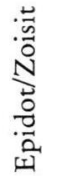 & $\begin{array}{l}\text { 苞 } \\
\text { J }\end{array}$ & 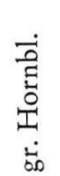 & 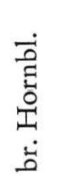 & 点 & 罠寻 & 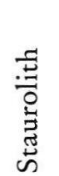 & 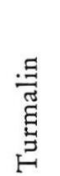 & 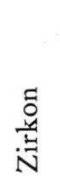 & $\begin{array}{l}\text { Gewichts- } \% \\
\text { der Gesamt- } \\
\text { probe }\end{array}$ \\
\hline
\end{tabular}

$2-40 \mathrm{~cm}$

$\begin{array}{lllllllllllllll}0,02-0,06 \mathrm{~mm} \varnothing & 47 & 1 & 8 & 1 & 11 & 21 & 9 & 5 & 4 & 1 & 1 & 3 & 35 & 0,55\end{array}$

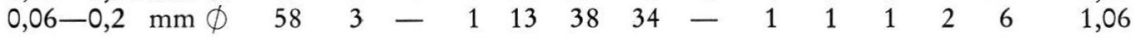

$40-50 \mathrm{~cm}$

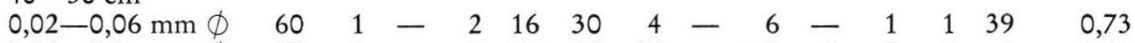

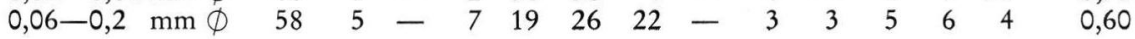


über den liegenden Sedimenten besitzt. Leider ließen sich in allen näher untersuchten polnischen Profilen keine sicheren Spuren des Laacher Bimstuffes aus der Allerödzeit nachweisen, so daß das Alter der Deckschicht nur als spätglazial angegeben werden kann (post-Vergletscherung, präholozän).

Ahnliche Profile sind auch auf den älteren Ablagerungen der Weichsel-Eiszeit zu finden, sie sind m. E. sogar typisch für viele Warthe-Ablagerungen. Zu berücksichtigen ist, daß der äolische Anteil in der Deckschicht ziemlich schwanken kann. Dabei zeigt sich wiederum eine deutliche Parallele zum Deckschutt der Mittelgebirge: Wachsender Schluffgehalt bedeutet oft Zunahme der „vulkanischen“ Schwerminerale und stärkere Verbraunung des Solums. Bei sehr geringem Schluffgehalt ist schwermineralogisch kein signifikanter Unterschied zum Liegenden festzustellen und kein $B_{v}$-Horizont, sondern ein fahler $\mathrm{A}_{1}$-Horizont über der Steinsohle ausgebildet. Als Beispiel sei ein Profil aus der Kiesgrube Kuczbork-Osada westlich von Mlawa aus dem Warthe-Bereich angeführt. Es liegt unter einem $5^{\circ}$ geneigten Nordhang und gliedert sich in:

$$
\begin{array}{cl}
0-20 \mathrm{~cm} & A_{\mathrm{p}} \text {-Horizont } \\
20-40 \mathrm{~cm} & \begin{array}{l}
\text { hellbrauner, schluffiger Sand (A } \mathrm{A}_{1} \text {-Horizont) an der Basis eine Lage } \\
\text { von Geröllen (eingeregelt) }
\end{array} \\
40-100 \mathrm{~cm} & \text { rostbrauner Sand mit dichter Ton-Bänderung (IIB }{ }_{\mathrm{t}} \text {-Horizont) } \\
100-120 \mathrm{~cm}+ & \text { hellgrauer Grobsand mit rostbraunen, tonigen Bändern. }
\end{array}
$$

Der Schluffgehalt ist über der Steinlage zwar deutlich höher als im Liegenden, jedoch insgesamt wesentlich geringer als im Allensteiner Profil (Tab. 3). In der Schwermineralführung bedeutet das Vorkommen einer braunen Hornblende in der Deckschicht allenfalls

\begin{tabular}{|c|c|c|c|c|c|c|c|c|c|c|c|c|c|c|}
\hline Flurabstand & 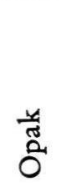 & $\frac{\text { 荡 }}{\frac{\pi}{\pi}}$ & 茄 & $\begin{array}{l}\text { 苟 } \\
\text { 营 }\end{array}$ & 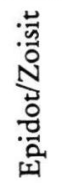 & 㫕 & $\begin{array}{l}\dot{0} \\
\dot{E} \\
\text { 它 } \\
\dot{\text { D. }}\end{array}$ & 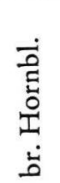 & 㐏 & 离 & 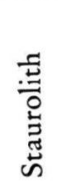 & $\frac{\Xi}{\Xi}$ & 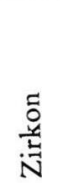 & $\begin{array}{l}\text { Gewichts-\% } \\
\text { der Gesamt- } \\
\text { probe }\end{array}$ \\
\hline
\end{tabular}
einen gewissen Hinweis auf äolische Beimengung (Tab. 4).

Tab. 3: Korngrößen (in \%) des Profils Kuczbork-Osada

\begin{tabular}{rccccccc}
\hline Flurabstand & $<0,002$ & $0,002-0,006$ & $0,006-0,02$ & $0,02-0,06$ & $0,06-0,2$ & $0,2-0,6$ & $0,6-2 \mathrm{~mm} \phi$ \\
\hline $20-40 \mathrm{~cm}$ & 2,0 & 2,8 & 5,9 & 7,9 & 6,0 & 53,9 & 19,3 \\
$40-100 \mathrm{~cm}$ & 3,3 & 1,7 & 0,1 & 1,5 & 2,6 & 53,6 & 29,9 \\
$100-120 \mathrm{~cm}$ & 0,6 & 0,2 & 0,7 & 1,6 & 1,7 & 37,0 & 58,0
\end{tabular}

Tab. 4: Schwermineralführung im Profil Kuczbork-Osada

$20-40 \mathrm{~cm}$

$\begin{array}{lllllllllrlrrrr}0,02-0,06 \mathrm{~mm} \phi & 61 & - & - & 1 & 28 & 26 & 2 & 1 & 10 & 1 & 1 & 1 & 29 & 0,16 \\ 0,06-0,2 \mathrm{~mm} \phi & 56 & 1 & - & 1 & 21 & 40 & 23 & - & 1 & 1 & 2 & 4 & 6 & 1,03 \\ 40-100 \mathrm{~cm} & & & & & & & & & & & & & & \\ 0,02-0,06 \mathrm{~mm} \phi & 75 & 1 & - & 1 & 19 & 16 & 8 & - & 11 & 1 & 1 & 2 & 40 & 0,44 \\ 0,06-0,2 \mathrm{~mm} \phi & 66 & 1 & - & 2 & 19 & 35 & 15 & - & 2 & 1 & 7 & 11 & 7 & 0,55\end{array}$

Die äolische Beeinflussung wird vor allem auch in Profilen auf Geschiebemergel deutlich. Hier läßt sich oft eine Zunahme des Sandgehaltes in der Deckschicht erkennen. Ein solches Profil ist z. B. durch den Hohlweg auf der flachen Kuppe ca. ein Kilometer südsüdöstlich des Dorfes Zelechowo (Selchow) nördlich Chojna (Königsberg/Neumark) freigelegt. Dieses Gebiet gehört zur kuppigen Grundmoräne des Pommerschen Stadiums. 


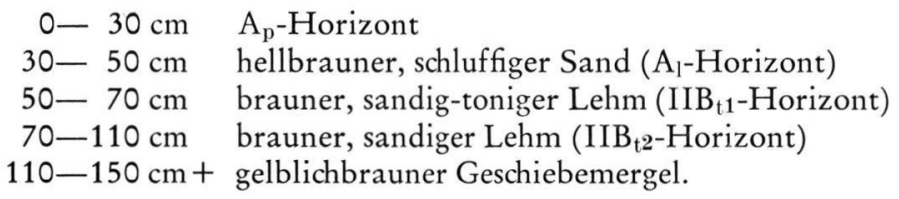

Wenn auch in diesem Profil keine Steinlage ausgebildet ist, so fällt doch bereits bei der Fingerprobe im Gelände der deutlich höhere Feinsandgehalt auf (Tab. 5), der sich nicht als Ergebnis einer Bodenbildung interpretieren läßt. Leider bleibt aber hier - und das ist durchaus keine Seltenheit — die Schwermineralführung im gesamten Profil annähernd

Tab. 5: Korngrößen (in \%/0) des Profils südlich Selchow

\begin{tabular}{rccccccc}
\hline Flurabstand & $<0,002$ & $0,002-0,006$ & $0,006-0,02$ & $0,02-0,06$ & $0,06-0,2$ & $0,2-0,6$ & $0,6-2 \mathrm{~mm} \phi$ \\
\hline $0-30 \mathrm{~cm}$ & 3,8 & 2,8 & 7,8 & 10,8 & 27,1 & 28,6 & 13,2 \\
$30-50 \mathrm{~cm}$ & 6,4 & 3,2 & 5,2 & 13,5 & 33,0 & 24,5 & 14,8 \\
$50-70 \mathrm{~cm}$ & 22,9 & 4,2 & 6,7 & 9,8 & 19,9 & 19,7 & 13,7 \\
$70-110 \mathrm{~cm}$ & 17,5 & 6,0 & 7,0 & 10,4 & 21,4 & 20,9 & 13,5 \\
$110-150 \mathrm{~cm}$ & 12,0 & 4,9 & 10,1 & 14,0 & 22,5 & 20,9 & 14,0
\end{tabular}

gleich, so daß von daher eine äolische Beeinflussung des Oberbodens nicht bewiesen werden kann. Die starke Zunahme der Kornoberflächen-Mattierung im Oberboden (von 5 auf $32 \%$ gibt gleichwohl einen Hinweis darauf, daß eine äolische Komponente in den Oberboden eingearbeitet wurde. Will man diese Einmischung nicht als kryoturbaten, sondern als bioturbaten, holozänen Vorgang deuten, so bleibt ungeklärt, weshalb in steinhaltigen Oberböden auf Hängen eine hangparallele Einregelung der Steine dominiert. Die Bioturbation müßte doch eine solche Einregelung verhindern. Dagegen bereitet eine Erklärung keine Schwierigkeiten, die davon ausgeht, daß periglaziale Solifluktion zwar auch mit kryoturbater Solimixion verbunden ist, die Solifluktion jedoch als vorherrschender Prozeß immer wieder die Einregelung der groben Komponenten bewirkt (vgl. die Untersuchungen an rezenten periglazialen Solifluktionsdecken bei SEMmEL 1969; Bibus et al. 1976). Die Solifluktion als holozänen Vorgang deuten zu wollen ist wenig überzeugend, wenn Messungen zu diesem Problem berücksichtigt werden, die ergeben haben, daß auf mitteleuropäischen Hängen solche Verlagerungen flächenhaft allenfalls bis in $10 \mathrm{~cm}$ Tiefe stattfinden (GöBel 1977). Die von Rohdenburg und Mitarbeitern (u. a. RohdenBURG 1978: 469) beschriebenen mittel- oder jungholozänen Schuttdecken sind m. E. als überwiegend reliefbedingte Sonderfälle zu deuten (z. B. starke Hangneigung). Es kann wohl ausgeschlossen werden, daß auf Hängen mit Neigungen gegen $2^{\circ}$ und sehr sandigem Untergrund unter holozänen Klimabedingungen solifluidale Verlagerungen weitflächig möglich sind.

Die Diskussion solcher Fragen erübrigte sich, wenn die Verknüpfung der Deckschichten mit kryoturbaten Formen (Tropfböden, Eiskeilen etc.) zu beobachten wäre. Solche Erscheinungen sind aus dem Weichselmoränen-Gebiet seit längerem bekannt und auch aus dem Gebiet des Pommerschen Stadiums beschrieben worden (z. B. Kliewe \& Schultz 1970). In manchen der von mir untersuchten Aufschlüsse waren zwar durchaus entsprechende Formen ausgebildet, jedoch ließ sich nirgends zweifelsfrei der Nachweis führen, $\mathrm{da}$ die oberste Deckschicht noch von diesen Prozessen mit erfaßt wurde.

Ein solches Profil ist z. B. nördlich von $\mathrm{km} 102$ unmittelbar westlich der Straße Bärwalde-Küstrin nördlich von Sarbinowo (Zorndorf) aufgeschlossen (Abb. 2). Dort liegt unter der braunen schluffigen Deckschicht ein an der Oberkante verlehmter Geschiebemergel des Frankfurter Stadiums. Die Deckschicht stimmt zugleich mit dem $\mathrm{A}_{1}$-Horizont 


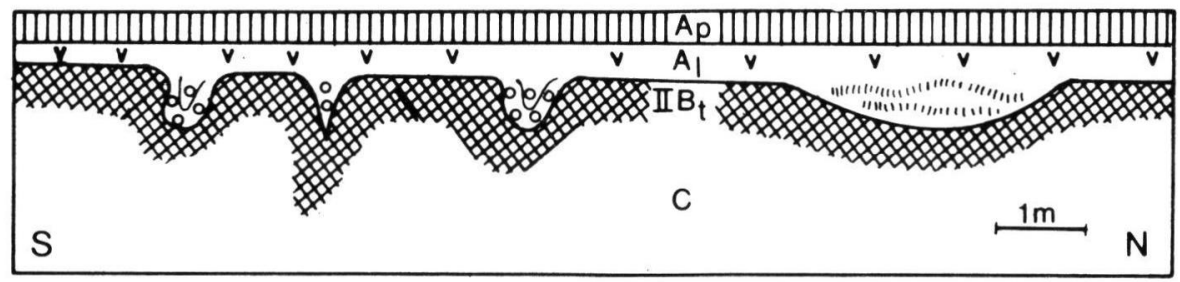

Abb. 2: Aufschluß nördlich Zorndorf. Der C-Horizont besteht aus Geschiebemergel, der an der Oberkante verlehmt ist (II $\mathrm{B}_{\mathrm{t}}$-Horizont). Darüber liegt eine sandig-schluffige Deckschicht $\left(\mathrm{A}_{1}\right.$ Horizont). Kryoturbate Taschen, Eiskeilpseudomorphosen und Erosionsrinnen zwischen der Deckschicht und dem Geschiebemergel sind mit kiesigem Sand gefüllt. Weitere Erläuterungen im Text.

der Parabraunerde überein, deren $\mathrm{IIB}_{\mathrm{t}}$-Horizont im Mergel entwickelt ist. Zwischen dieser Deckschicht und dem Mergel lag jedoch ursprünglich ein kiesiger Sand, von dem Reste in Kryoturbationstaschen, Eiskeilpseudomorphosen und Erosionsrinnen erhalten sind. Im Gegensatz zur hangenden Deckschicht wurden diese Sedimente noch von den kryoturbaten Verformungen erfaßt. Die spätere Verlehmung des Mergels zeichnete die Umrisse der periglazialen Formen nach. In den sandigen Füllungen bildeten sich braune Tonbänder.

Eiskeilpseudomorphosen sind auch in Kiesgruben der Sander des Pommerschen Stadiums zu finden, so etwa südöstlich von Soldin (Neumark) in einer Kiesgrube südlich von Trzcinna. Dort ist die Deckschicht ein brauner, schwach kiesiger schluffiger Sand, der in $50 \mathrm{~cm}$ Tiefe von einer glatt durchziehenden Steinlage mit schlecht ausgeprägten Windkantern abgeschlossen wird. Darunter folgt ein $20 \mathrm{~cm}$ starker hellbrauner Sand, der die Spalten von ehemaligen Eiskeilpolygonen ausfüllt. Diese greifen in den liegenden Sanderkies hinein. Auch hier wird also wieder deutlich, daß die Eiskeile v or der Bildung der Deckschicht aktiv gewesen sein müssen. Im übrigen zeigt das Vorkommen der Deckschicht auf Sandern, daß die Existenz einer Deckmoräne i. S. von Solger (zuletzt 1965; vgl. auch Grimmel 1973: 24) nicht unbedingte Voraussetzung für die Entstehung periglazialer Deckschichten sein muß.

Die bisher nicht beobachtete Verknüpfung von periglazialen Frostmusterformen mit der Deckschicht widerlegt nicht deren periglazialen Charakter. Auch aus anderen Gebieten, etwa dem Rhein-Main-Gebiet, sind Aufschlüsse bekannt, in denen ältere jungpleistozäne Sedimente kryogen gestört wurden, dagegen jungtundrenzeitliche nicht, obwohl diese an benachbarten Orten eindeutige kryoturbate Störungen aufweisen. In diesem Zusammenhang sei aber erwähnt, daß laut JERSAK (1975) in äolischen Ablagerungen dieses Alters in Polen keine echten Eiskeile mehr zu finden sind. Beobachtungen von KOZARSKI (1972) und Churska (1972) belegen die Existenz von Eiskeil-Pseudomorphosen im Jungmoränengebiet Nord-Polens. KozArski (1974: 80 f.) betont indessen, daß in Nordwest-Polen bisher keine Periglazialstrukturen gefunden wurden, die mit Sicherheit der Jüngeren Tundrenzeit zugerechnet werden könnten (vgl. jedoch CHURSKA, ib.: 69).

\section{Vergleich mit älteren und jüngeren Sedimenten}

Es wurde bereits betont, daß auf vielen Ablagerungen der Warthe-Zeit ähnliche Deckschichten liegen. Gute Aufschlüsse sind außer bei Mlawa noch im Sandergebiet östlich Lodz (westlich Brezany) und östlich Zawonia (östlich Trebnitz) zu finden. Gleichwohl ist nicht zu bestreiten, daß gerade mit dem Löß der Trebnitzer Umgebung eine drastische Abweichung gegeben ist. Dieses Gebiet zeigt immer wieder Profile, in denen über Geschiebemergel, der als warthezeitlich angesehen wird, Weichsellöß liegt, der in der Ziegelei Treb- 
nitz zwei bis drei Meter mächtig ist und den Lohner Boden (Mittelwürm) enthält. Interglaziale Böden wurden nicht gefunden. Auf Deckschichtenbildung im Sandlöß westlich dieses Gebietes geht bereits KowaLKowsKi (1967) ein.

Weshalb an einer Stelle stark gegliederte und mächtigere Deckschichten auf den warthezeitlichen Sedimenten liegen, an anderen Orten dagegen nur Substrate, die den Deckschichten auf weichselzeitlichen Sedimenten gleichen, kann - die stratigraphische Einordnung als richtig vorausgesetzt - seine Ursache darin haben, daß im letzten Fall bis in die späte Weichselzeit die Abtragung dominierte oder die Sedimentation so gering war, daß in der spätglazialen Deckschicht die vorher abgelagerten Substrate aufgearbeitet wurden. Verschiedene Autoren betonen, daß die periglaziale Deckserie im DDR-Gebiet auf den Jungmoränen ähnlich der auf den Altmoränen ist (z. B. LemKe 1972: 72). Nur im Bereich des Pommerschen Stadiums sei die Intensität der periglazialen Prozesse etwas geringer gewesen.

Die Entkalkungstiefe ist nach meinen Beobachtungen auf warthezeitlichen Geschiebemergeln eindeutig größer als auf weichselzeitlichen. Auch bei kalkfreien Sanden und Kiesen reicht die bodengenetisch bedingte Tonbänderung in den älteren Ablagerungen oft deutlich tiefer, jedoch ist auf weichselzeitlichen Kiesen stellenweise gleichfalls eine Bänderung bis drei Meter unter Flur anzutreffen. In dieser Hinsicht wird also eine Unterscheidung der verschieden alten Sedimente schwierig. Doch sei betont, daß in der DDR Befunde für eine bodengeographische Grenze entlang der Pommerschen Endmoräne vorliegen (u. a. HaAse \& Schmidt 1975: 198). Ein eindeutiger Unterschied zwischen dem Aufbau des hier diskutierten Deckschichten-Typs in $\mathrm{n}$ - und a u ß erhalb des Gebietes des Pommerschen Stadiums scheint mir in Polen zumindest an vielen Stellen nicht nachweisbar. Auch von polnischer Seite wird des öfteren die deutliche periglaziale Formung $\mathrm{nach}$ dem Pommerschen Stadium betont. So beschreibt z. B. KozARsKi (1974) periglaziale Hangsedimente, die aus verschiedenen Kalt-Phasen des Spät-Würms stammten, die sämtlich jünger als die Pommersche Phase seien. In Übereinstimmung mit Untersuchungsbefunden aus der DDR (u. a. LEMKE 1972) sind gut ausgebildete Windkanterlagen im Gebiet des Pommerschen Stadiums allem Anschein nach selten zu finden. Es sei aber darauf hingewiesen, daß von polnischen Autoren (z. B. KozArski 1978: 302) periglaziale Dünen in besonders großem Ausmaß als Bildungen der Jüngeren Tundrenzeit erkannt wurden. Jedoch wird angenommen, daß zu dieser Zeit die im Vergleich zu früheren kalten Perioden schon besser entwickelte Vegetation die Entstehung größerer Dünen wegen der stärkeren Hindernisbildung für den Treibsand gefördert habe.

\section{Schriftenverzeichnis}

Bibus, E., Nagel, G. \& Semmel, A. (1976): Periglaziale Reliefformung im zentralen Spitzbergen. - Catena, 3: 387-398; Gießen.

Blume, H. P., Hofmann, R. \& Pachur, H.-J. (1978): Periglaziäre Steinring- und Frostkeilbildungen norddeutscher Parabraunerden. - Mitt. deutsch. bodenkdl. Ges., 27: 345-346; Göttingen.

Chunska, Z. (1972): Periglaziale Umbildung der Jungmoränengebiete (im Gebiet des Wisla- und Dreweca-Urstromtales). - Wiss. Z. E.-M.-Arndt-Universität Greifswald, Jg. XXI: 69-70; Greifswald.

Diemann, R. (1977): Ausbildung und Entstehung des Substrattyps Tieflehm im nördlichen Jungmoränengebiet der DDR. - Z . geol. Wiss., 5: 1275-1276; Berlin.

GöBEL, P. (1977): Vorläufige Ergebnisse der Messung gravitativer Bodenbewegungen auf bewaldeten Hängen im Taunus. - Catena, 3: 387-398; Gießen.

Grimmel, E. (1973): Bemerkungen zum Geschiebedecksand. - Eiszeitalter u. Gegenwart, 23/24: 16-25; Öhringen. 
HaAse, G. \& Schmidt, R. (1975): Struktur und Gliederung der Bodendecke der DDR. - Peterm. geogr. Mitt., 119. Jg: 279-300; Gotha/Leipzig.

Jersak, J. (1975): Frost Fissures in Loess Deposits. - Biuletyn Periglacialny, 24: 245-258; Lodz.

Kliewe, H. \& Schultz, H.-J. (1970): Die periglaziäre Fazies im Jungmoränengebiet nördlich der pommerschen Eisrandlage. - Peterm. geogr. Mitt., Erg. H. 274: 255-263; Gotha/Leipzig.

Kopp, D. (1965): Die periglaziäre Deckzone (Geschiebedecksand) im nordostdeutschen Tiefland und ihre bodenkundliche Bedeutung. - Ber. geol. Ges. DDR, 10: 739-771; Berlin.

- (1970): Kryogene Perstruktion und ihre Beziehung zur Bodenbildung im Moränengebiet. Peterm. geogr. Mitt., Erg. H. 274: 169-279; Gotha/Leipzig.

- \& JÄGER, K.-D. (1972): Das Perstruktions- und Horizontprofil als Trennmerkmal periglaziärer und extraperiglaziärer Oberflächen im nordmitteleuropäischen Tiefland. - Wiss. Z. E.-M.Arndt-Univ. Greifswald, XXI. Jg.: 77-84; Greifswald.

Kowalkowski, A. (1967): Eigenschaften und Entstehung der Böden auf Sandlöß in den Dalkauer Bergen im Katzengebirge. - Albr.-Thaer-Arch., 11: 483-501; Berlin.

KozarsKI, S. (1972): Beweise des Spätwürm-Dauerfrostboden-Vorkommens im Bereiche der letzten Vereisung Nordwestpolens und deren paläogeographische Bedeutung. - Wiss. Z. E.-M.Arndt-Univ. Greifswald, XXI. Jg.: 67-68; Greifswald.

- (1974): Evidences of Late-Würm Permafrost Occurence in North-West Poland. - Quaestiones Geographicae, 1: 65-86, Poznań.

- (1978): Das Alter der Binnendünen in Mittelwest-Polen. - Beiträge zur Quartär- und Landschaftsforschung, Festschr. J. Fink: 291-305; Wien.

Lembke, H. (1972): Die Periglazialerscheinungen im Jungmoränengebiet der DDR. - Wiss. Z. E.-M.-Arndt-Univ. Greifswald; XXI. Jg.: 71-76; Greifswald.

LiedtKe, H. (1975): Die nordischen Vereisungen in Mitteleuropa. - Forsch. deutsch. L.-Kde., 204: 160 S., 1 farb. Kte. 1 : 1000 000; Bonn-Bad Godesberg.

Rohdenburg, H. (1978): Zur Problematik der spätglazialen und holozänen Bodenbildung in Mitteleuropa. - Beiträge zur Quartär- und Landschaftsforschung, Festschr. J. Fink: 467471; Wien.

Semmel, A. (1964): Junge Schuttdecken in hessischen Mittelgebirgen. - Notizbl. hess. L.-Amt Bodenforsch., 92: 275-285; Wiesbaden.

- (1968): Studien über den Verlauf jungpleistozäner Formung in Hessen. - Frankf. geogr. Hefte, 45: 133 S.; Frankfurt a. M.

- (1969): Verwitterungs- und Abtragungsvorgänge in rezenten Periglazialgebieten (Lappland und Spitzbergen). - Würzb. geogr. Arb., 26: 82 S.; Würzburg.

- (1973): Periglaziale Umlagerungszonen auf Moränen und Schotterterrassen der letzten Eiszeit im deutschen Alpenvorland. - Z. Geomorph. N. F., Suppl. Bd. 17: 118-132; Berlin/ Stuttgart.

Solger, F. (1965): Zur methodischen Behandlung der Geschiebedecksandfrage. - Ber. geol. Ges. DDR, 10: 727-738; Berlin. 